\section{Arbuscular Mycorrhiza Influences Growth and Nutrient Uptake of Asparagus (Asparagus officinalis L.) under Heat Stress}

\author{
Rumana Yeasmin ${ }^{1}$ \\ School of Biological, Earth, and Environmental Sciences and Ecology and \\ Evolution Research Centre, University of New South Wales, Sydney, NSW \\ 2052, Australia; Hawkesbury Institute for the Environment, Western Sydney \\ University, Richmond, NSW 2753, Australia; and The United Graduate \\ School of Agricultural Sciences, Tottori University, 4-101 Koyama-Minami, \\ Tottori 680-8553, Japan \\ Stephen P. Bonser \\ School of Biological, Earth, and Environmental Sciences and Ecology and \\ Evolution Research Centre, University of New South Wales, Sydney, NSW \\ 2052, Australia

\section{Satoru Motoki} \\ Faculty of Agriculture, Meiji University, 1-1-1, Higashi-Mita, Tama-Ku, \\ Kawasaki-Shi, Kanagawa 214-8571, Japan

\section{Eiji Nishihara \\ The United Graduate School of Agricultural Sciences, Tottori University, 4-101 Koyama-Minami, Tottori 680-8553, Japan}

Additional index words. arbuscular mycorrhizal fungi, asparagus, Glomus intraradices, growth response, heat stress, nutrient uptake

\begin{abstract}
Environmental conditions, specifically heat stress, are important factors in asparagus crop production. Arbuscular mycorrhizal fungi (AMF) have been shown to increase plant growth. Effects of heat stress on nutrient uptake have rarely been examined in intact plants, but the limited results indicate that heat stress will decrease uptake; no studies have examined heat stress effects on asparagus nutrient uptake. We examined the effects of AMF, Glomus intraradices, on the growth, nutrient uptake, heat stress responses, and antioxidative activity in asparagus (Asparagus officinalis L.). We grew AMF-inoculated or non-AMF-inoculated asparagus plants in sand culture at 20 to $25^{\circ} \mathrm{C}$ for 14 weeks in a greenhouse and subsequently subjected to three temperature conditions (control $=20^{\circ} \mathrm{C} /$ $25^{\circ} \mathrm{C}$ night $/$ day, mild heating $=30{ }^{\circ} \mathrm{C} / 35^{\circ} \mathrm{C}$ night $/$ day, and severe heating $=37^{\circ} \mathrm{C} / 42{ }^{\circ} \mathrm{C}$ night/day) in growth chambers. Morphological and physiological growth parameters were compared between AMF-inoculated and non-AMF-inoculated plants. The mycorrhizal symbiosis markedly enhanced biomass production and heat stress responses negatively in plants compared with that in the non-AMF-inoculated plants. Plants grown under nonAMF-inoculated treatment had severe rate of leaf browning $(80 \%$ to $100 \%)$, whereas the mycorrhizal plants showed a minimum rate of leaf browning under heat stress conditions. The results indicated mycorrhizal-inoculated plants showed an increase activity of antioxidative enzymes, such as superoxide dismutase and ascorbate peroxidase. The 2,2-diphenyl1picrylhydrazyl radical scavenging activity also showed a greater response in mycorrhizal plants than in the control plants under each temperature treatment. Application of AMF enhanced plant growth and mineral nutrients and alleviated heat stress damage through an increased antioxidative activity and the mycorrhizal symbiosis significantly enhanced heat stress tolerance of asparagus.
\end{abstract}

Received for publication 18 Sept. 2018. Accepted for publication $11 \mathrm{Feb} .2019$.

This study was funded by the Japanese Government MEXT (Ministry of Education, Culture, Sports, Science and Technology, or "Monbukagakusho") scholarship program.

${ }^{1}$ Corresponding author. E-mail: yeasminbd@gmail. com.
Asparagus (Asparagus officinalis L.) is a well-managed/assisted crop, with good water and nutrient availability (Yeasmin et al., 2013). Asparagus decline is associated with both abiotic and biotic factors. Abiotic factors associated with the decline of asparagus production are related with the release of allelopathic compounds, including nutrient imbalance, and deterioration of physical and chemical conditions. Improving biotic conditions to promote asparagus growth was recently attempted by inoculation of beneficial microbes, such as AMF that had a high affinity for asparagus, and the symbiotic relationship promotes vigor in asparagus plants (Nahiyan and Matsubara 2012). Traditionally, asparagus has been grown in temperate climates, but in the past few years, commercial production of asparagus has begun in tropical climates. Thus, asparagus appears to have a wide environmental adaptability. To date, the growth and physiological responses of asparagus of high temperature has not been well researched.

Heat stress often decreases the uptake of nutrients in plant tissues or decreases the total content of nutrients in the plants, although effects can vary among nutrients and species (Giri et al., 2017). Hungria and Kaschuk (2014) reported that heat stress can also disrupt enzymes involved in nutrient metabolism (e.g., nitrate and ammonium assimilation). When a plant undergoes heat stress, several toxic reactive oxygen species (ROS) are generated in cells, and the oxidative stress caused by these ROS is one of the major damaging factors in plants (Wahid et al., 2007). To limit oxidative damage under stress conditions, plants have developed a series of detoxification systems involving several antioxidative enzymes, such as superoxide dismutase (SOD), ascorbate peroxidase (APX), peroxidase, catalase, or glutathione reductase (Zhu et al., 2010). The mechanisms underlying the influence of AMF on the ROS metabolism of host plants under heat stress conditions remain uncertain.

AMF are well known to increase plant growth and yield, improve water and nutrient uptake, and to lessen abiotic stress factors (Mouk and Ishii, 2006). AMF establish mutualistic symbioses with most higher plants and benefit plants by increasing resistance to environmental stresses, enhancing plant nutrient acquisition, and improving soil quality (Hart et al., 2018). As a result of the increased nutrient uptake, plants inoculated with mycorrhizae frequently produce higher yields than do those without AMF. Moreover, AMF inoculations into the plantations of soybean, core, millet, trifoliate orange, rice, and 17 tropical legumes have been demonstrated with improved economic impact on agriculture and horticulture by enhancing plant growth and yield (Gholamhoseini et al., 2013). For these reasons, mycorrhizae can play an important role in agricultural production by creating the possibility of enhancing absorption of nutrients. Mycorrhizae particularly enhance $\mathrm{P}$ uptake but can also increase $\mathrm{NH}_{4}{ }^{+}$and $\mathrm{NO}_{3}{ }^{-}$uptake, as well as the uptake of other nutrients, including $\mathrm{Zn}$, $\mathrm{Cu}$, and $\mathrm{K}$ (Hart et al., 2018). However, it has been unclear how antioxidative factors change through symbiosis with AMF in asparagus plants and how the changes are associated with heat tolerance. We hypothesized that 1) AMF will increase growth and 
nutrient uptake of the affected heat-stressed asparagus plants, 2) the increased activity of enzymatic antioxidants in mycorrhizal asparagus imply that the AMF symbiosis can alleviate ROS damage, protect plants against oxidation, and improve heat stress tolerance during plant production. Testing these hypotheses will extend our present understanding of plant responses to heat stress by examining the importance of AMF under the recent advent of global warming.

\section{Materials and Methods}

Plant materials, growth conditions, and AMF inoculation. Asparagus seed variety 'Gijnlim' of European origin, was purchased from a local commercial seed company in Japan. Sandy soil used in this experiment was first sterilized at $121{ }^{\circ} \mathrm{C}$ for $15 \mathrm{~min}$ in an automatic high-pressure steam sterilized autoclave (MLS-2420; Sanyo, Tokyo, Japan). Fresh pregerminated 20-d-old seedlings of 'Gijnlim' were sown in sandy soil-filled pots $\left(790 \mathrm{~cm}^{3}\right)$. Plants were initially grown for 12 weeks in a greenhouse at 20 to $25{ }^{\circ} \mathrm{C}$ with $14 \mathrm{~h}$ of daylight and $60 \%$ to $70 \%$ relative humidity. After transferring to a growth chamber (MLR-351H; Sanyo), the plants were grown for 4 weeks with $12 \mathrm{~h}$ of light at $25^{\circ} \mathrm{C}$ (day and night), 400 to $500 \mu \mathrm{mol} \cdot \mathrm{m}^{-2} \cdot \mathrm{s}^{-1}$ photosynthetically active radiation, and $60 \%$ relative humidity. The seedlings were then inoculated with AMF (3 g/plant) inoculum for mycorrhizal plants and an equal amount of autoclaved inoculum for the nonmycorrhizal (control) plants. A commercial inoculum was used (Idemitsukosan Co. Ltd., Tokyo, Japan), containing Glomus intraradices (spore density unknown). The mycorrhizal and autoclaved inocula were placed at $4 \mathrm{~cm}$ below the transplanted seedlings.

Temperature treatments and harvesting. Following inoculation, plants were randomly assigned for three temperature treatments for 4 months (light and photoperiod as described previously): control $=20{ }^{\circ} \mathrm{C} / 25{ }^{\circ} \mathrm{C}$ (night $/$ day), mild heating $=30^{\circ} \mathrm{C} / 35^{\circ} \mathrm{C}$ (night/day), and severe heating $=37^{\circ} \mathrm{C} / 42^{\circ} \mathrm{C}$ (night/day). Day and night temperatures were both raised the same number of degrees during heat treatments, since climate-change projections indicate that global warming will affect both days and nights (and, in fact, will increase night, more than day, temperatures on average. Our mild heat treatment was intended to mimic a severe heat wave under today's climate, whereas the severe heat treatment was intended to mimic a severe heat wave in future climate predictions, following significant global warming. Irrigation was done at $50 \mathrm{~mL} \cdot \mathrm{d}^{-1}$ per pot; fertilization immediately after each planting, and then at 15-d intervals with $100 \mathrm{~mL}$ of a solution containing $10 \mathrm{mg} \cdot \mathrm{L}^{-1}$ of $\mathrm{N}\left(\mathrm{NH}_{4} \mathrm{Cl} 1.8 \mathrm{mg} \cdot \mathrm{L}^{-1}+8.2 \mathrm{mg} \cdot \mathrm{L}^{-1} \mathrm{HNO}_{3}\right)$, $8 \mathrm{mg} \cdot \mathrm{L}^{-1} \mathrm{P}, 27 \mathrm{mg} \cdot \mathrm{L}^{-1} \mathrm{~K}, 4.0 \mathrm{mg} \cdot \mathrm{L}^{-1} \mathrm{Ca}, 0.1$ $\mathrm{mg} \cdot \mathrm{L}^{-1} \mathrm{Mn}$, and $0.1 \mathrm{mg} \cdot \mathrm{L}^{-1} \mathrm{~B}$. Harvesting was done at the end of 4 months of growth in the growth chamber. After harvesting, all seedlings were carefully separated into roots and shoots, thoroughly cleaned, blotted dry between absorbing paper and their measurements were done according to the following steps. All treatments were replicated four times $(\mathrm{n}=4$ per treatment, per harvest).

Measurement of AMF colonization. Four plants per treatment with four replications were used to determine the percentage of AMF colonization. AMF colonization levels of roots were determined at 4 months after AMF inoculation (under control, mild, and severe heat stress condition). The lateral roots (abbreviated RFCSL) were cut into $1-\mathrm{cm}$ fragments and preserved in a solution of formalin:glacial acetic acid:ethanol $(5: 5: 50 \mathrm{v} / \mathrm{v} / \mathrm{v})$. Before examination, fragments were rinsed with tap water and heated in $10 \%(\mathrm{w} / \mathrm{v}) \mathrm{KOH}$ for $12 \mathrm{~h}$ at $45{ }^{\circ} \mathrm{C}$. After a thorough rinse with water, samples were stained in acid fuchsin (Liu et al., 2007). AMF colonization was assessed using the 20. magnified intersections method (Nahiyan and Matsubara 2012).

Plant growth and heat stress symptoms. The dry weight of shoot and root of the four plants per treatment were assessed under control and heat stress conditions after ovendrying at $100{ }^{\circ} \mathrm{C}$ for $24 \mathrm{~h}$. In addition, plant height, total number of leaves/plants, maximum size of the leaves/plant, and total number of roots/plants were measured, and leaf browning, as a heat stress symptom in plants, was determined. The leaves of the plants were divided into three groups: 1) whole green leaves, 2) half-browned leaves, and 3) fully browned leaves. Subsequently, the percentage of leaf browning per plant was calculated, and the rate of leaf browning was categorized into five groups: 1) $0 \%$ (no browning); 2) less than $20 \%$ browning; 3 ) $20 \%$ to $40 \%$; 4) $40 \%$ to $80 \%$; and 5) $80 \%$ to $100 \%$ browning.

Nutrient uptake measurements. Nutrient uptake was estimated from the dry biomass of shoots of 12 plants per treatment. All dry leaves and shoots were combined and ground to a fine powder using a stainless ball mill, and analyzed for nutrient uptake following Yeasmin et al. (2013). Dried samples of $50 \mathrm{mg}$ of leaves and stems were pulverized, and total $\mathrm{N}$ content was determined by the dry combustion method with an automated C-N coder (Model MT 700; Yonaco, Tokyo, Japan). Total $\mathrm{K}$ was determined after digestion with a $\mathrm{H}_{2} \mathrm{O}_{2}-\mathrm{H}_{2} \mathrm{SO}_{4}$ mixture. Briefly, dried samples of $100 \mathrm{mg}$ were also pulverized and digested by wet ashing via dissolution in $5 \mathrm{~mL}$ sulfuric acid and $4 \mathrm{~mL}$ hydrogen peroxide and heating at $80^{\circ} \mathrm{C}$ for $6 \mathrm{~h}$. Total $\mathrm{P}$ in the digested mixture was determined calorimetrically with a spectrophotometer (Model U-2001, Hitachi Co., Tokyo, Japan) using the phosphomolybdate blue method (Yeasmin et al., 2013). The total amounts of $\mathrm{K}$ were determined with an atomic absorption spectrophotometer (Model Z-8100, polarized Zeeman; Hitachi Co., Tokyo, Japan) after dilution in distilled water and filtration through a glass fiber filter (GA-100; Advantec Co., Ltd., Tokyo, Japan).

Analysis of antioxidative abilities. The antioxidative enzyme activities of SOD and APX, nonenzymatic antioxidant compounds (ascorbic acid and polyphenol) and the total antioxidant activity based on DPPH (2,2- diphenyl-1-picrylhydrazyl) radical scavenging activity were analyzed twice under control and heat stress conditions. The enzymes were extracted using $0.15 \mathrm{~g}$ of plant sample in $3 \mathrm{~mL}$ of $50 \mathrm{~mm}$ phosphoric acid buffer solution, $\mathrm{pH}$ 7.0. The extract was centrifuged at 12,000 rpm for $12 \mathrm{~min}$ at $5{ }^{\circ} \mathrm{C}$, and the activity of both SOD and APX was analyzed in the supernatant. Analysis of antioxidative enzyme activities and antioxidative substances were carried out according to the methods of Nahiyan and Matsubara (2012) (SOD), and $\mathrm{Wu}$ et al. (2006) (APX) (DPPH radical scavenging activity), respectively. Briefly, the total SOD activity was measured by the ability of SOD to inhibit the reduction of nitro blue tetrazolium (NBT) through photochemically generated superoxide radicals. Absorbance was recorded at $560 \mathrm{~nm}$, and one unit of SOD was defined as the amount of enzyme required to inhibit the reduction rate of NBT by $50 \%$ at $25{ }^{\circ} \mathrm{C}$. The APX activity was estimated as the reduction in the absorbance due to ascorbic acid at $290 \mathrm{~nm}$. The reaction was initiated on the addition of $0.1 \mathrm{~mm} \mathrm{H}_{2} \mathrm{O}_{2}$, and a reduction in the absorbance was measured for $60 \mathrm{~s}$ at $290 \mathrm{~nm}$ in a spectrophotometer (Model U-2001; Hitachi Co., Tokyo, Japan). Antioxidants were extracted from plant samples using the same procedure as that used to extract the enzymes, except that different extraction buffers were used. The DPPH radical scavenging activity test was performed by extracting plant samples with ethanol at a ratio of $0.15 \mathrm{~g} / 3 \mathrm{~mL}$, and the extracts $(150 \mu \mathrm{L})$ were mixed with $2.7 \mathrm{~mL}$ of a $20 \%$ ethanol solution of DPPH. After incubating for $30 \mathrm{~min}$, the absorbance of the samples was read at $520 \mathrm{~nm}$ using a spectrophotometer (Model U-2001; Hitachi Co.).

Statistical analysis. Dry weight (4 months after AMF inoculation) was analyzed by Tukey's multiple range test at $P \leq 0.05$. Mean values were separated by $t$ test for dry weight, RFCSL, and antioxidative abilities at $P \leq 0.05$. All analyses were performed using XLSTAT pro statistical analysis software (Addinsoft, New York, NY).

\section{Results}

AMF root colonization. Microscope observations confirmed that the noninoculated plants were not colonized by $G$. intraradices. Percentage mycorrhiza colonization showed a tendency to be higher in the control condition than in the heat-stressed conditions. There were no significant differences in root colonization among the three stress treatments (Fig. 1).

Effects of AMF inoculation on plant growth. Heat stress (both mild and severe) decreased both shoot and root dry biomass, and root growth was more sensitive than shoot growth under severe heat stress (Fig. 2). Vigorous growth was observed in mycorrhizal asparagus plants compared with that of the nonmycorrhizal control plants under both temperature (mild and severe) conditions. AMF-inoculated plants had greater shoot and root dry biomass than control plants under the control treatment. Plant growth 


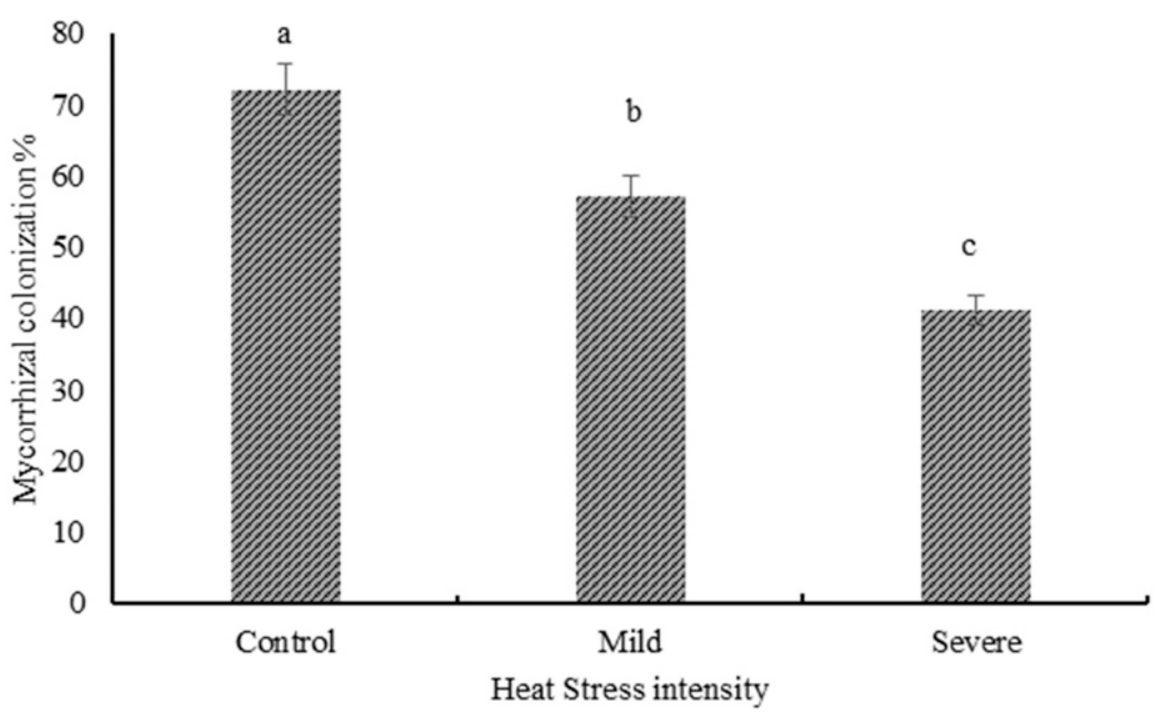

Fig. 1. Mycorrhizal root colonization rate of asparagus under control $=20{ }^{\circ} \mathrm{C} / 25^{\circ} \mathrm{C}$ (night $/$ day), mild $=$ $30{ }^{\circ} \mathrm{C} / 35^{\circ} \mathrm{C}$ (night/day), and severe $=37^{\circ} \mathrm{C} / 42^{\circ} \mathrm{C}$ (night/day) heat stress conditions $(\mathrm{n}=4)$ of AMF inoculation, respectively. The data represent the means, and the lines indicate the SE. Different lowercase letters indicate significant difference ( $t$ test, $P \leq 0.05)$ among heat stress treatments.

decreased under heat stress conditions, but shoot dry weight remained significantly greater in AMF-inoculated plants compared with that in control plants; differences in root dry biomass were also significant (Fig. 2). Symptoms of temperature stress, such as narrow and yellowing leaves, were observed in asparagus plants under heat stress intensity. Leaf injury, recorded as the level of leaf browning (in percentage), was less severe in the AMFinoculated plants under heat stress (Fig. 3).

Nutrient uptake. The uptake rate of macronutrients $(\mathrm{N}, \mathrm{P}$, and $\mathrm{K})$ and the micronutrients $(\mathrm{Ca}, \mathrm{Mg}$, and $\mathrm{Fe})$ by shoots and leaves decreased with increasing heat stress (Fig. 4). After 4 months of heat stress, the levels of nutrient uptake in moderately stressed and severely stressed plants were less than unheated controls. Compared with plants in the control temperature treatment, plants in the heat stress treatments were more affected in the uptake of $\mathrm{N}, \mathrm{P}, \mathrm{K}$, and other nutritional elements. In addition, the effect of mycorrhizal treatment was significant on all parameters related to nutritional status. The results demonstrated that AMF-inoculated plants accumulated more nutrients when compared with the noninoculated plants grown under different heat stress intensity. In this study, C:N ratio in the shoot and root tended to be less in heat-stressed plants relative to control treatment, whereas root $\mathrm{C}: \mathrm{N}$ ratio was more affected than shoot growth under severe heat stress (Fig. 5).

Antioxidative abilities in plants. The AMF symbiosis significantly increased SOD activity in the shoot and roots under both control and heat stress (both mild and severe) conditions compared with the control plants (Fig. 6). Significantly different APX activities were observed in the shoot and roots between mycorrhizal and control plants under control, mild, and severe heat stress conditions. Concomitantly, the DPPH radical scavenging activity was also increased in mycorrhizal plants as compared with that in controls under both temperature conditions.

\section{Discussion}

AMF-inoculated asparagus plants grew better than the non-AMF-inoculated mycorrhizal plants in the presence and absence of heat stress. An increased expression of different antioxidants in mycorrhizal asparagus plants compared with that in non-AMFinoculated plants under control and heat stress treatments was observed in the present study. Temperature stress can affect the growth of plants and development of the mycorrhizal symbiosis (Zhu et al., 2010). Low temperatures influence AMF development, whereas high temperatures affect AMF activity and have a negative or no effect on mycorrhizal colonization (Martin and Stutz, 2004). In the present study, heat stress had a negative effect on mycorrhizal colonization. In contrast, the highest colonization rate was obtained in inoculated plants that were not subjected to any heat stress. This is most likely because the contents of the mycorrhizal fungi are infected by the host plants under stressful conditions (Evelin et al., 2009). Positive effects from mycorrhizal inoculation are attributed to improvements in the uptake of nutrients (Mouk and Ishii, 2006). These results were also observed by Diallo et al. (2001) in cowpea plants inoculated with AMF.

Growth. Mycorrhizal colonization, improved growth and dry matter in asparagus exposed to various intensities of heat stress (Fig. 2). Previous researchers have found that the efficiency of AMF increases with increasing stress intensity (Matsubara et al., 2000). In the present experiment, dry mass of roots increased by mycorrhiza inoculation in all temperature stress treatments. Many factors affect plant

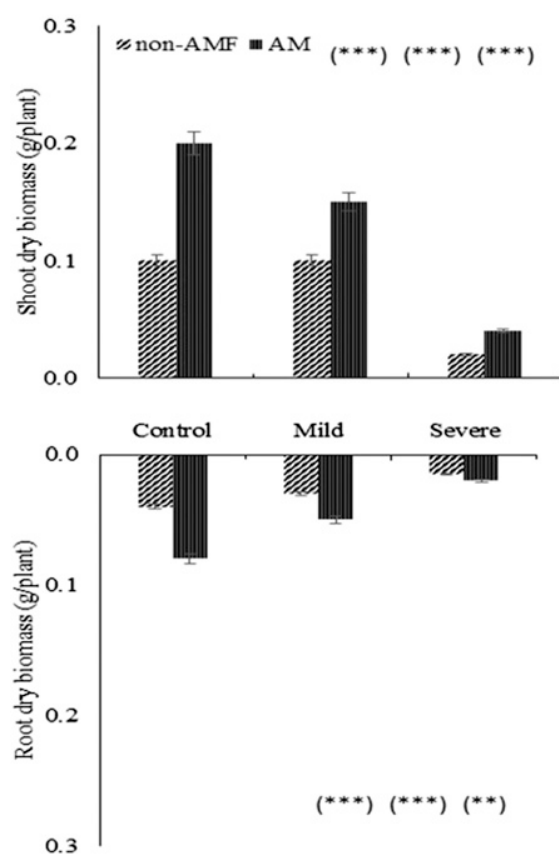

Fig. 2. Dry weight of shoots and roots of asparagus plants after arbuscular mycorrhizal fungus (AMF) Glomus intraradices inoculated and non-AMFinoculated plants under control $=20{ }^{\circ} \mathrm{C} / 25^{\circ} \mathrm{C}$ (night/day), mild $=30{ }^{\circ} \mathrm{C} / 35^{\circ} \mathrm{C}$ (night/day), and severe $=37{ }^{\circ} \mathrm{C} / 42{ }^{\circ} \mathrm{C}$ (night/day) heat stress conditions, respectively. The data represent the means, and the lines indicate the SE. Within each variable, the significance of different heat stress (control, mild and severe) effect is indicated in parentheses $(* P<0.05, * * P<0.01, * * * P<$ $0.001, \mathrm{NS}=$ not significant $)$

growth responses to AMF, including environmental conditions, AMF identity, and diversity. Some studies found that abiotic stress could enhance mycorrhizal responses, whereas opposite results were also reported. For example, salt stress increased growth response of maize to Glomus mosseae (Sheng et al., 2008). In agreement with the present study, Zhu et al. (2010) showed that mycorrhizal plants had greater root dry weight than nonmycorrhizal plants at low temperature stress. Similar results were observed by Martin and Stutz (2004), who found pepper (Capsicum annum L.) shoot and root dry weight affected by interaction between mycorrhiza and temperature stress. This result is consistent with data from studies in maize (Zhu et al., 2010) showing that mycorrhizal symbiosis can affect the growth of plants under different environmental temperatures. Heat stress has been reported as one of the most important causes of reductions in yield and dry biomass production in many crop plants (Wahid et al., 2007).

Nutrient uptake. High temperatures and radiation limit plant productivity through direct effects on biological processes, and temperature also influences microbes involved in nutrient uptake and substrate mineralization (Martin and Stutz, 2004). High-temperature stress reduces root growth, number, and mass (Huang et al., 2012), which affects the growth of aboveground tissue by restricting the supply of water and mineral nutrients, affecting 

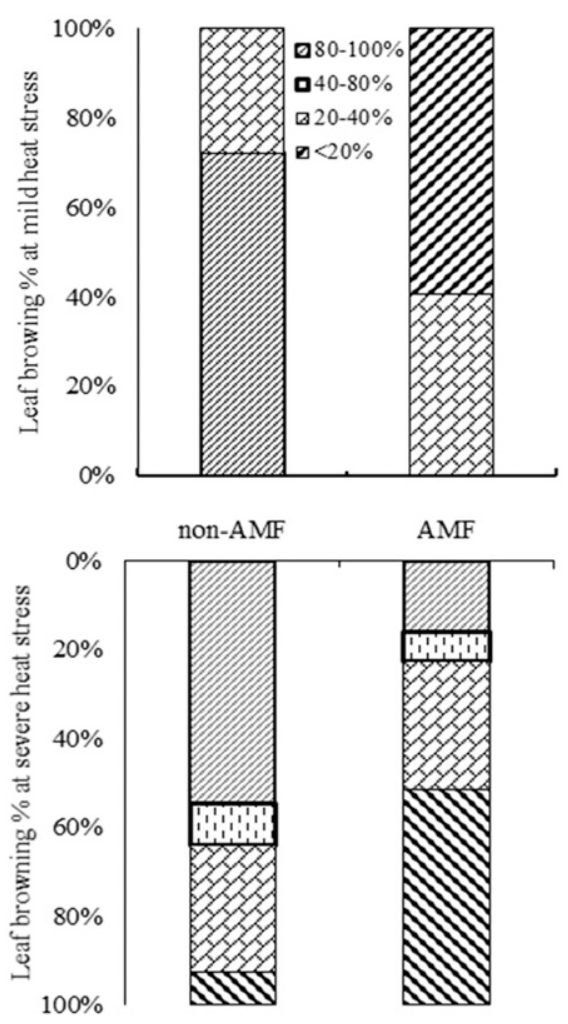

Fig. 3. Percentage leaf browning in asparagus after arbuscular mycorrhizal fungus (AMF): Glomus intraradices inoculated and non-AMF-inoculated plants; under control $=20^{\circ} \mathrm{C} / 25^{\circ} \mathrm{C}$ (night/day), mild $=30{ }^{\circ} \mathrm{C} / 35^{\circ} \mathrm{C}$ (night/day), and severe $=$ $37{ }^{\circ} \mathrm{C} / 42{ }^{\circ} \mathrm{C}$ (night/day) heat stress conditions, respectively.

production of hormones synthesized in roots and transported to shoots (Wahid et al., 2007). Several studies have demonstrated that plants colonized by AMF are much more efficient in taking up soil $\mathrm{P}$ than noninoculated plants (Gholamhoseini et al., 2013). There are various possible mechanisms by which $P$ availability and absorption can be enhanced through the application of AMF: 1) by enhancing the P uptake that is facilitated by the extensive hyphae of the fungus, allowing them to explore more soil volume than can the nonmycorrhizal plants (Evelin et al., 2009); and 2) by increasing the microbial biomass. Microbial biomass increases when mycorrhizal fungi are added to the soil, increasing the release of $\mathrm{CO}_{2}$, which, in turn forms $\mathrm{H}_{2} \mathrm{CO}_{3}$ in the soil solution. The resulting weak acid can dissolve primary P-containing minerals, thereby increasing $\mathrm{P}$ availability. It has also been reported that $\mathrm{P}$ concentrations in the leaves may affect the stomatal response to environmental stress, perhaps by affecting the energetics involved in guard cell osmotic parameters or by wall stiffening governing the stomatal movements (Gholamhoseini et al., 2013). Thus, it is not surprising that plants inoculated with mycorrhizal fungi show higher performance when compared with noninoculated plants, even under stressful conditions.

$\mathrm{K}$ plays a key role in plant metabolism, particularly under stressful environment
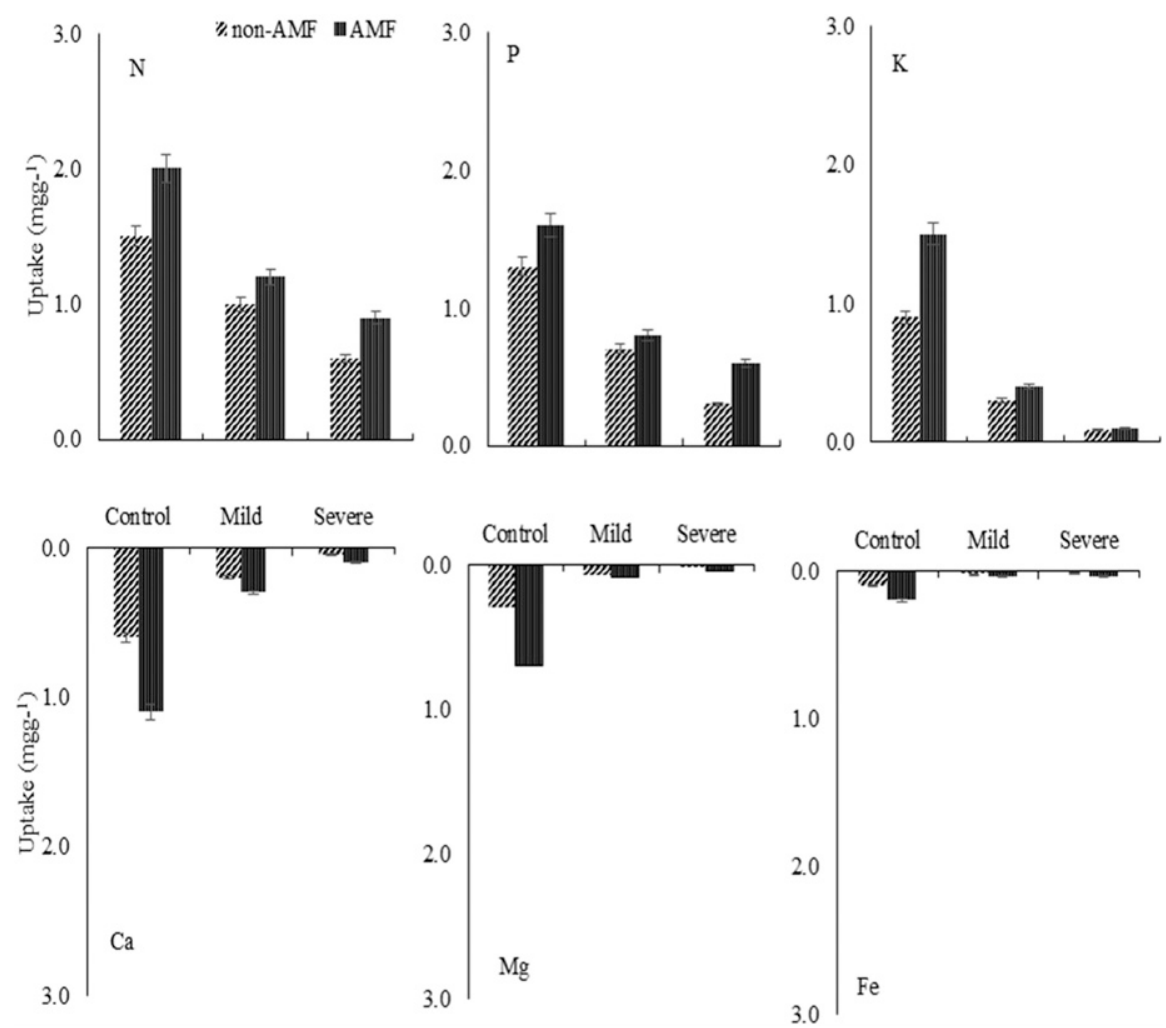

Fig. 4. Mycorrhizal dependency of nutrient uptake under control, mild, and severe heat stress conditions $(n=4)$. The data represent the means, and the lines indicate the SE. Within each variable, the significance of different heat stress (control, mild, and severe) effect is indicated in parentheses $\left({ }^{*} P<\right.$ $0.05, * * P<0.01, * * * P<0.001, \mathrm{NS}=$ not significant) within arbuscular mycorrhizal fungus (AMF) inoculation, and non-AMF-inoculated plants.

(Gholamhoseini et al., 2013). K activates a range of enzymes, and plays an important role in stomatal movements and protein synthesis (Evelin et al., 2009), and it has been reported that mycorrhizal colonization can enhance $\mathrm{K}$ absorption by plants (Giri et al., 2017). Increased $K$ concentration can be a consequence of the increased $P$ availability from mycorrhizal fungal activity. In addition Gholamhoseini et al. (2013) found a synergistic relationship between $\mathrm{P}$ and $\mathrm{K}$, in which increased $\mathrm{P}$ availability in the root zone increased $\mathrm{K}$ absorption in plants. Our results show that plants inoculated with mycorrhizal fungi show higher nutrient uptake $(\mathrm{N}, \mathrm{P}, \mathrm{K}, \mathrm{Ca}, \mathrm{Mg}$, and $\mathrm{Fe})$ compared with that in nonmycorrhizal plants under stressful conditions (Fig. 4). Decreases in nutrient uptake per unit root might be caused by depletion of labile $\mathrm{C}$ (total nonstructural carbohydrate), and hence energy, in roots (e.g., due to a decrease in transport of shoot $\mathrm{C}$ to roots or an increase in root respiration) or by direct heat damage to roots (Huang et al., 2012).

Antioxidative abilities in plants. Temperature stress induces the production of ROS and causes physiological disorders in plants (Wahid et al., 2007). Tolerance to heat stress in crop plants has been associated with an increase in antioxidant enzyme activity (Zhu et al., 2010). Some reports described that AMF colonization itself induced a temporary increase in antioxidative abilities, such as SOD, guaiacol peroxidase, catalase, APX, and flavonoid content, suggesting that colonization might be temporary stress for host plants ( $\mathrm{Wu}$ et al., 2006). In this study, SOD, APX, and DPPH radical scavenging activity in both root and shoot increased in all the AMF plants (Fig. 6). This is consistent with previous observations on the leaves and roots of citrus seedlings colonized by Glomus versiforme during drought (Wu et al., 2006), and leaves and roots of maize plants colonized by Glomus etunicatum during temperature stress ( $\mathrm{Zhu}$ et al., 2010). In the aspects of such antioxidative factors, our results support their findings. SOD acts as a defensive reaction and detoxifies $\mathrm{O}_{2}^{-}$among the antioxidative enzymes; thus, SOD activity is considered the most important key enzyme in antioxidative abilities in plants (Nahiyan and Matsubara, 2012). Our results showed similar patterns to those findings because of the increase in SOD activity related to heat stress tolerance.

Several analytical methods are routinely used to evaluate the capacity of antioxidant compounds in biological systems (Wu et al., 2006; Zhu et al., 2010). DPPH radical scavenging activity is a rapid, simple, and inexpensive method to measure antioxidant capacity in biological compounds and involves the use of the free-radical DPPH (Marxen et al., 2007). The analyses of asparagus plants under control and heat stress conditions revealed a mycorrhizal-induced DPPH radical scavenging activity that is 


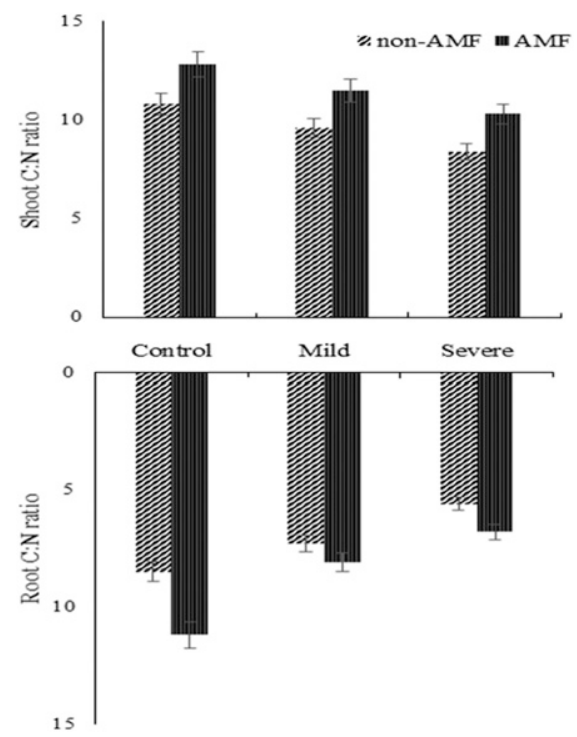

Fig. 5. C:N ratio of arbuscular mycorrhizal fungus (AMF)-inoculated and non-AMF-inoculated asparagus plants under severe, mild, and no heat stress (control) conditions $(n=4)$. The data represent the means, and the lines indicate the SE.

concomitant with the enhancement of antioxidative activity by the mycorrhizal symbiosis. Increased DPPH radical scavenging activity has likewise been reported in mycorrhizal asparagus under biotic stress (Nahiyan and Matsubara, 2012). Although AMF symbiosis affects antioxidant production, the exact mechanisms involved remain unclear.

The present study, which aimed to investigate the effects of different AMF symbiosis on growth and nutritional status of asparagus, has yielded invaluable knowledge on the physiological and morphological responses that are known to be among the most effective in association with heat stress. The increased activity of enzymatic antioxidants and higher production of nonenzymatic antioxidant compounds in mycorrhizal asparagus imply that the AMF symbiosis can alleviate ROS damage, protect plants against oxidation, and improve heat stress tolerance during plant production. The inoculation of AMF led to significant increases in root and shoot dry mass and asparagus $\mathrm{N}, \mathrm{P}$, and $\mathrm{K}$ content in comparison with non-AMF-inoculated plants. Therefore, we conclude that the AMF should be preferred for use in asparagus production as a beneficial growth activator, especially in areas in which symptoms of $\mathrm{N}, \mathrm{P}, \mathrm{K}$, or other element deficiencies predominate under adverse climatic conditions, thus contributing to minimize asparagus decline.

\section{Literature Cited}

Diallo, A.T., P.I. Samb, and H. Roy-Macauley. 2001. Water status and stomatal behaviour of cowpea, Vigna unguiculata L. Walp, plants inoculated with two Glomus species at low soil moisture levels. Eur. J. Soil Biol. 37:187-196.
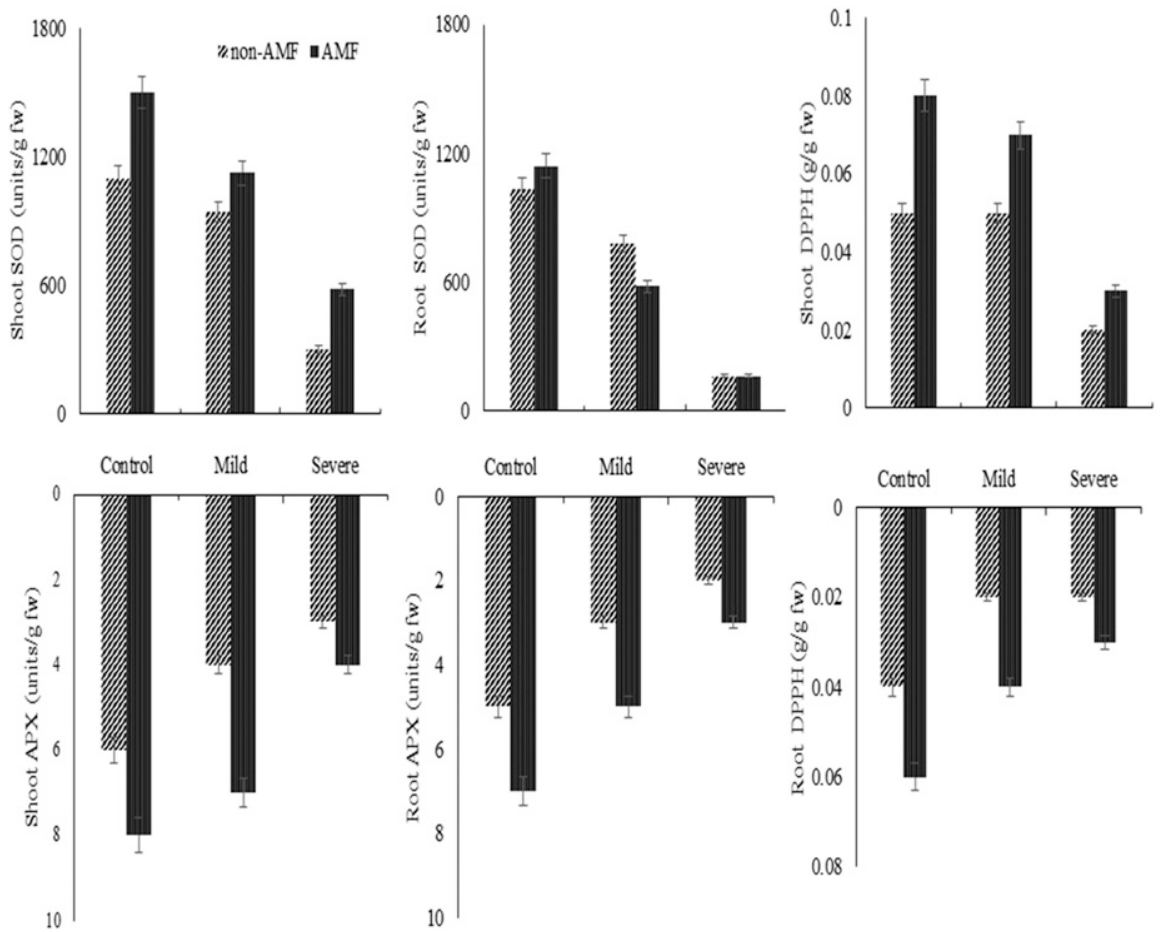

Fig. 6. Mycorrhizal dependency of superoxide dismutase (SOD), ascorbate peroxidase (APX), and 2,2diphenyl-1-picrylhydrazyl (DPPH) activity in the shoots and roots of asparagus after arbuscular mycorrhizal fungus (AMF): Glomus intraradices inoculated and non-AMF-inoculated asparagus; under control $=20^{\circ} \mathrm{C} / 25^{\circ} \mathrm{C}$ (night/day), mild $=30{ }^{\circ} \mathrm{C} / 35^{\circ} \mathrm{C}$ (night/day), and severe $=37^{\circ} \mathrm{C} / 42{ }^{\circ} \mathrm{C}$ (night/day) heat stress conditions $(n=4)$, respectively.

Evelin, H., R. Kapoor, and B. Giri. 2009. Arbuscular mycorrihizal fungi in alleviation of salt stress: A review. Ann. Bot. 104:1263-1280.

Gholamhoseini, M., A. Ghalavand, A. Dolatabadian, E. Jamshidi, and A. Khodaei-Joghan. 2013. Effects of arbuscular mycorrhizal inoculation on growth, yield, nutrient uptake and irrigation water productivity of sunflowers grown under drought stress. Agr. Water Mgt. 117:106-114.

Giri, A., S. Heckathorn, S. Mishra, and C. Krause. 2017. Heat stress decreases levels of nutrientuptake and -assimilation proteins in tomato root plants. Plants 6:1-15.

Hart, M.M., P.M. Antunes, V.B. Chaudhary, and L.K. Abbott. 2018. Fungal inoculants in the field: Is the reward greater than the risk? Funct. Ecol. 32:126-135.

Huang, B., S. Rachmilevitch, and J. Xu. 2012. Root carbon and protein metabolism associated with heat tolerance. J. Expt. Bot. 63:3455-3465.

Hungria, M. and G. Kaschuk. 2014. Regulation of $\mathrm{N}_{2}$ fixation and $\mathrm{NO}_{3} / \mathrm{NH}_{4+}$ assimilation in nodulated and $\mathrm{N}$-fertilized Phaseolus vulgaris L. exposed to high temperature stress. Environ. Exp. Bot. 98:32-39.

Liu, J., L. Wu, S. Wei, X. Xiao, C. Su, P. Jiang, Z. Song, T. Wang, and Z. Yu. 2007. Effects of arbuscular mycorrhizal fungi on the growth, nutrient uptake and glycyrrhizin production of licorice (Glycyrrhiza uralensis Fisch). Plant Growth Regulat. 52:29-39.

Martin, C.A. and J.C. Stutz. 2004. Interactive effects of temperature and arbuscular mycorrhizal fungi on growth, $\mathrm{P}$ uptake and root respiration of Capsicum annum L. Mycorrhiza 14:241-244.

Matsubara, Y., Y. Kayakawa, and H. Fukui. 2000. Temperature stress tolerance of asparagus seedlings through symbiosis with arbuscular mycorrhizal fungus. J. Jpn. Soc. Hort. Sci. 69:570-575.
Marxen, K., K.H. Vanselow, S. Lippemeier, R. Hintze, A. Ruser, and U.P. Hansen. 2007. Determination of DPPH radical oxidation caused by methanolic extracts of some microalgal species by linear regression analysis of spectrophotometric measurements. Sensors (Basel) 7:2080-2095.

Mouk, B.O. and T. Ishii. 2006. Effect of arbuscular mycorrhiza fungi on tree growth and nutrient uptake of Sclerocarya birrea under water stress, salt stress and flooding. J. Jpn. Soc. Hort. Sci. 75:26-31.

Nahiyan, A.S.M. and Y. Matsubara. 2012. Tolerance to fusarium root rot and changes in antioxidative ability in mycorrhizal asparagus plants. HortScience 47:356-360.

Sheng, M., M. Tang, H. Chen, B. Yang, F. Zhang, and Y. Huang. 2008. Influence of arbuscular mycorrhizae on photosynthesis and water status of maize plants under salt stress. Mycorrhiza 18:287-296.

Wahid, A., S. Gelani, M. Ashraf, and M.R. Foolad. 2007. Heat tolerance in plants: An overview. Environ. Exp. Bot. 61:199-223.

Wu, Q.S., R.X. Xia, and Y.N. Zou. 2006. Reactive oxygen metabolism in mycorrhizal and nonmycorrhizal citrus (Poncirus trifoliata) seedlings subjected to water stress. J. Plant Physiol. 163:1101-1110

Yeasmin, R., F. Kalemelawa, S. Motoki, H. Matsumoto, K. Nakamatsu, S. Yamamoto, and E. Nishihara. 2013. Root residue amendment on varietal allelopathy and autotoxicity of replanted asparagus (Asparagus officinalis L.). Exp. Agr. Hort. 2:31-44.

Zhu, X., F. Song, and H. Xu. 2010. Influence of arbuscular mycorrhiza on lipid peroxidation and antioxidative enzyme activity of maize plants under temperature stress. Mycorrhiza 20:325-332. 